Evolution and official growth in housing of the Provincial Housing Institute

PALABRAS CLAVE • ARPUITECTURA RESIDENCIAL PALABRAS CLAVE - AROUITECTURA RESIDENCIAL . RESIDENCIAL VIVIENDA SOCIAL CRECIMIENTO OFICAL

KEYWORDS - RESIDENTIAL ARCHITECTURE - FLEXIBILITY IN HOUING . OFFICIAL GROWTH

\section{RESUMEN}

La vivienda no es un hecho estático con principio y final definidos, sino un proceso adaptable y en constante cambio. Por ello, el objetivo del trabajo que aquí se presenta es analizar el crecimiento oficial de las viviendas construidas por el Instituto Provincial de la Vivienda, durante la fase de uso, localizadas en la Provincia de San Juan. La metodología utilizada se basó, primeramente, en una investigación teórica bibliográfica sobre el tema, que permita caracterizar los antecedentes de la vivienda como proceso evolutivo e identificar sus particularidades. Luego, mediante imágenes satelitales y planos arquitectónicos, se analizó la tipología original y el crecimiento de 4 I viviendas. En el estudio se enfatizó en la ocupación del terreno, el aspecto funcional y tecnológico constructivo de las ampliaciones. Se concluye que es necesario incorporar espacios para nuevos usos e incrementar las dimensiones de los espacios sociales existentes. Además, controlar el crecimiento en relación con las dimensiones del terreno.

\section{ABSTRACT}

Housing is not a static event with a defined beginning and end, but an adaptable and constantly changing process. Therefore, the objective of the work is to analyze the official growth of the houses built by the Provincial Housing Institute, during the use phase, located in the Province of San Juan. The methodology used was based, first, on a theoretical bibliographic investigation on the subject, which allows characterizing the antecedents of housing as an evolutionary process and identifying its particularities. Then, using satellite images and architectural plans, the original typology and growth of 41 homes were analyed. The study emphasized the ocupation of the land, the functional and constructive technological aspect of the extensions. It is concluded that, it is necessary to incorporate spaces for new uses and increase the dimensions of the existing social spaces. In addition, to control the growth in relation to the dimensions of the land.

\title{
Evolución y crecimiento oficial en viviendas del Instituto Provincial de la Vivienda
}

\author{
MARÍA CELINA MICHAUX: https://orcid.org/0000-0003-0347-6402 · Universidad Nacional de San Juan \\ celinamichaux@faud.unsi.edu.ar \\ GRACIELA MELISA VIEGAS: https://orcid.org/0000-0001-6248-4678 · Universidad Nacional de La Plata \\ gachiviegas@yahoo.com.ar \\ IRENE ALICIA BLASCO LUCAS: https://orcid.org/0000-0002-1326-895X · Universidad Nacional de San Juan \\ iblasco@faud.unsi.edu.ar
}

Fecha de recepción: 30 septiembre 2021 · Fecha de aceptación: 22 noviembre 2021

\section{INTRODUCCIÓN}

La vivienda es un bien indispensable para la reproducción social, alberga la familia, simboliza refugio y protección y si bien no existe un consenso general, su vida útil varía entre los 40 y 100 años (Camargo Sierra, 2019). Para Petrović et al. (2021), la vida útil está entre los 50 y 100 años y de modo similar, Ziemski (2018) la establece entre 40 y 60 años. En argentina, la Norma IRAM 11553 (1969), define la vida útil de una construcción como el período de tiempo que debe durar física y económicamente y la estima entre 30 y 50 años. En concordancia, la Secretaría de Vivienda (2019), establece 30 años como período de vida útil mínimo.

En el ciclo de vida de una vivienda, la fase de uso es la que ocupa mayor cantidad de tiempo, abarca desde el momento en que se entrega a su propietario hasta que deja de utilizarse. Durante esta etapa, las actividades del núcleo familiar y sus demandas espaciales influyen en la configuración arquitectónica de la misma, provocando modificaciones espaciales o constructivas en la tipología original (Lucas et al., 2012; Adler et al., 2018). Esto implica que la vivienda tiene una condición inherentemente progresiva, donde el usuario encuentra el modo de modificarla según sus necesidades para vivir más cómodamente, haciéndose partícipe de su crecimiento (Muñoz Lin, 2014; Teles y González, 2013). Según Agyefi-Mensah (2013), cualquier diseño o modificación en la vivienda debe ser abordada desde la actividad de los usuarios, considerando el efecto del tiempo. En esta línea, se entiende a los procesos de crecimiento como un hecho real y habitual, que se desarrolla con naturalidad en gran cantidad de viviendas.

Sin embargo, en base a entrevistas realizadas en el Instituto Provincial de la Vivienda (IPV), Dirección de Planeamiento y desarrollo Urbano (DPDU) y en la FAUD-UNSJ al momento, no se ha estudiado el desarrollo de viviendas construidas por el IPV, ni sus procesos de crecimiento. En general, se desconocen en su praxis, cantidad y calidad las modificaciones que se realizan en las mismas (A. Grgic; J. Cueli; A. Merlo comunicación personal, agosto de 2020). Por tanto, se especifica como pregunta de investigación; żcuáles son las características de las variaciones producidas durante los procesos de crecimiento en las viviendas IPV del Área Metropolitana de la Provincia de San Juan (AMSJ), construidas entre 1990 y 2000? ¿̇Qué formas tecnológico-constructivas se evidenciaron en las diferentes acciones de crecimiento oficial de las viviendas IPV del AMSJ?

Esta problemática cobra jerarquía considerando que el IPV, encargado de la planificación, diseño y construcción de viviendas estatales, ha edificado a la fecha 78.000 viviendas, las cuales representan el $44.2 \%$ del total en la provincia (FIGURA 1) (Instituto de Investigaciones Económicas y Estadísticas, 2020). Por esto, el 
1. Barrios construidos por el IPV en el AMSJ (izq.) y barrios IPV que forman parte del estudio (der.). Fuente: elaboración propia en base a IPV y Google Maps, 2021.

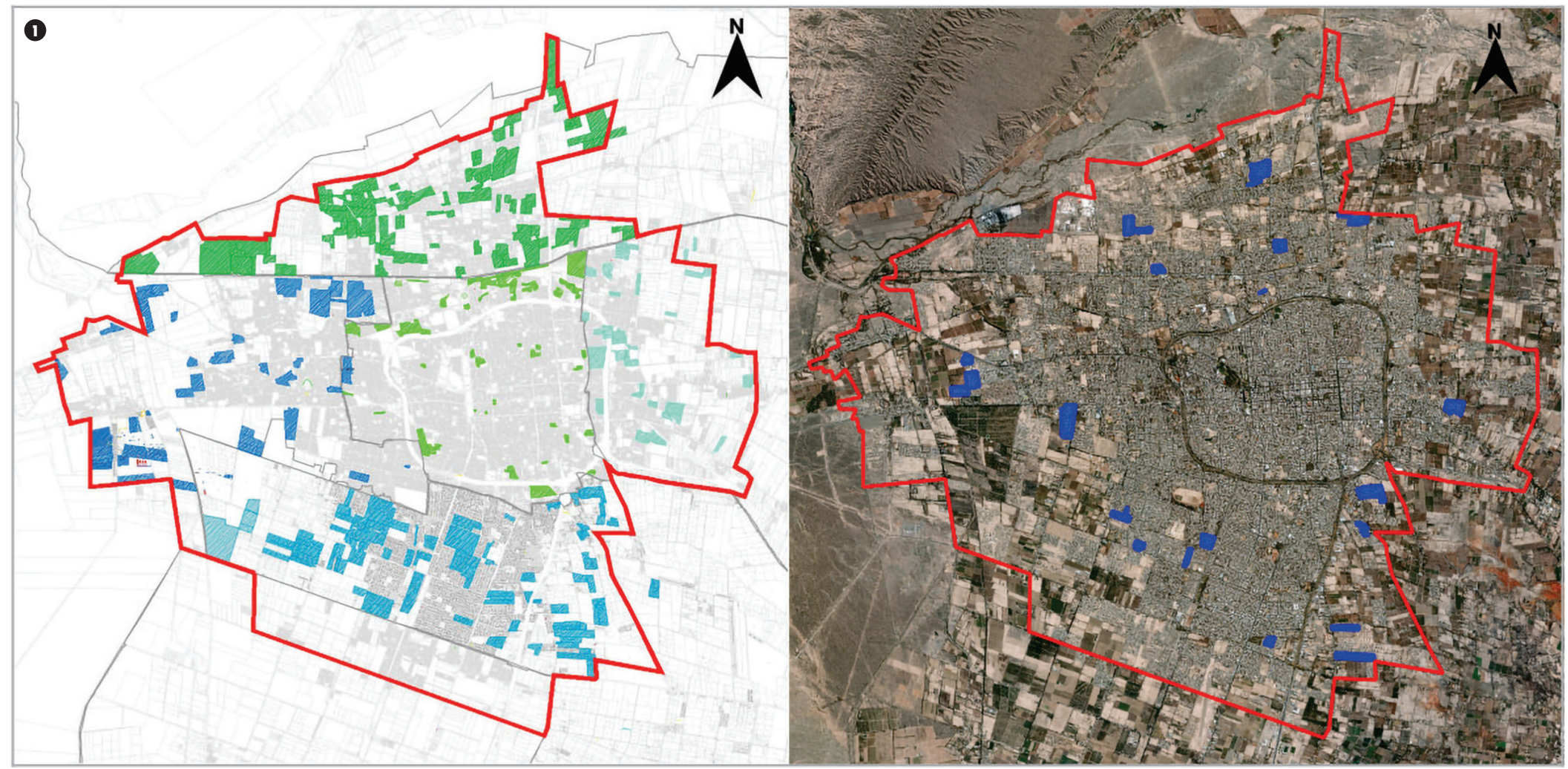

objetivo del trabajo es analizar la evolución temporal de las viviendas construidas por el IPV, durante la fase de uso, localizadas en la Provincia de San Juan.

\section{METODOLOGÍA}

La metodología se basa, en primer lugar, en una investigación teórico-descriptiva que busca caracterizar los antecedentes de la vivienda flexible e identificar sus particularidades, mediante revisión bibliográfica. La selección bibliográfica se realizó mediante metabuscadores, donde se filtraron los documentos (predominantemente artículos científicos) de los últimos 20 años y no se impusieron límites geográficos. Como técnica de recolección de datos, se utilizó la revisión de fuentes de observación secundaria y la sistematización de la información se realizó mediante fichajes. El procedimiento incluyó la organización, clasificación y análisis bibliográfico. Para la elaboración de las tablas se tomaron como criterios, la época y los aspectos conceptuales de mayor jerarquía.

Seguidamente, se realizó un análisis cuantitativo y gráfico de las ampliaciones oficiales de 41 viviendas (casos de estudio). Para estudiar los procesos de crecimiento oficiales, se utilizaron imágenes satelitales de libre disponibilidad y se obtuvieron los planos arquitectónicos de la vivienda original y en su estado actual, de los archivos de la DPDU. Como instrumentos de investigación se utilizaron fichas y gráficas comparativas de datos.

\section{CASOS DE ESTUDIO}

Para definir los casos de estudio, del total de barrios construidos por el IPV en la provincia, se seleccionaron aquellas viviendas individuales unifamiliares en planta baja, ubicadas dentro del AMSJ y construidas entre 1990 y 2000, es decir que llevan entre 20 y 30 años de uso y han alcanzado su vida útil mínima (FIGURA 1). Llegado este punto, se identificaron 3.790 viviendas, de 20 barrios, que cumplen estas condiciones. Seguidamente, se relevaron las viviendas ampliadas, mediante imágenes satelitales y del total de viviendas, se obtuvo que el $89.97 \%$ creció (3410 viviendas) y el 10.03\% mantuvo el prototipo original. Este análisis previo a la definición de los casos de estudio contextualiza la importancia de la temática en el AMSJ y aporta a una mejor comprensión del problema antes planteado. 
2. Barrios y viviendas que componen la muestra. Fuente: elaboración propia en base a Google Maps, 2021.
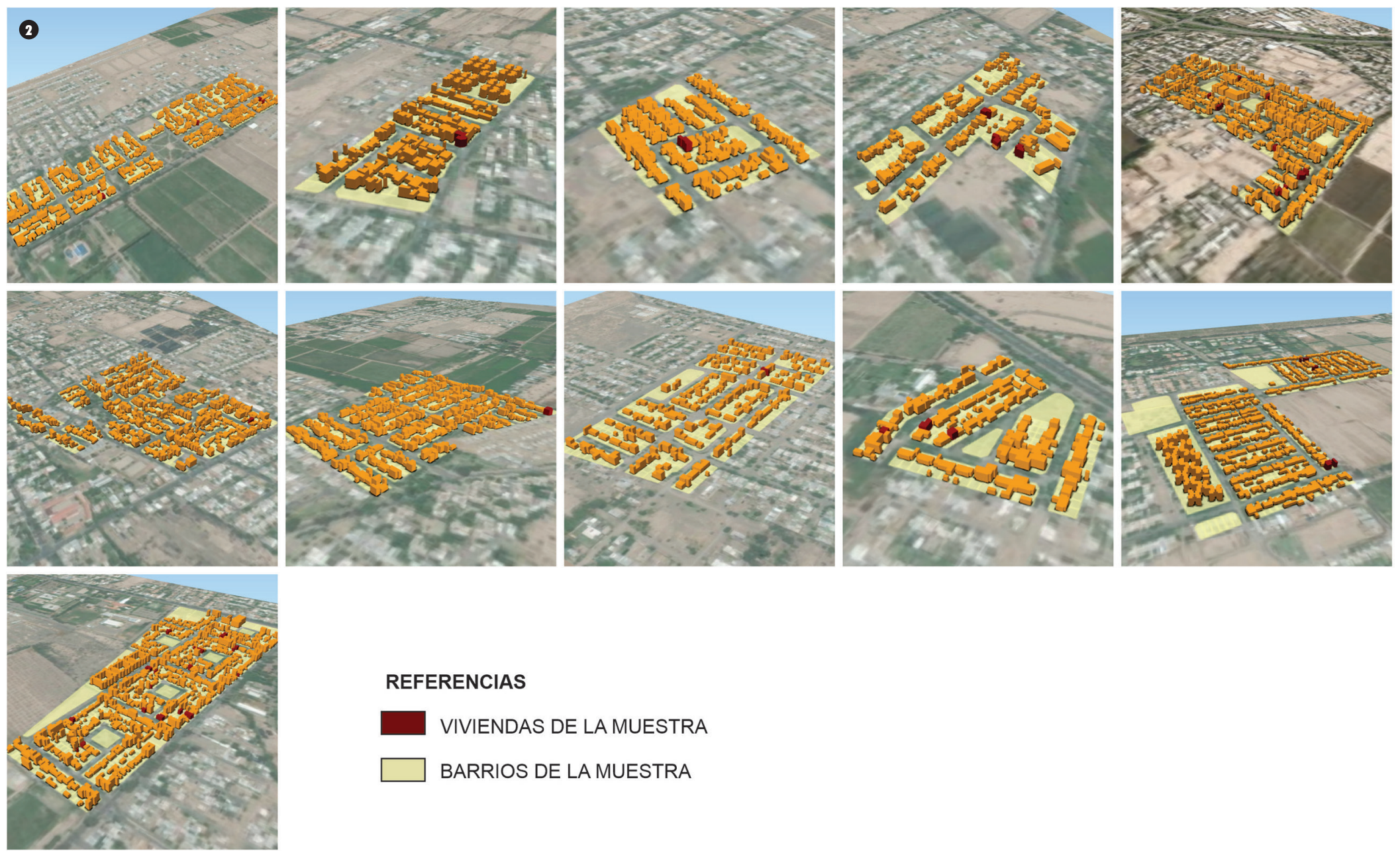

\section{REFERENCIAS}

VIVIENDAS DE LA MUESTRA

BARRIOS DE LA MUESTRA

Seguidamente, se buscaron en DPDU las viviendas ampliadas que realizaron los procesos de crecimiento de forma oficial, mediante la presentación de los planos de arquitectura. Se localizaron 72 expedientes y solo 45 con la información completa, legible y donde la ampliación se corresponde con el plano, por esto, se llega a que la muestra se compone por 41 viviendas pertenecientes a 11 barrios del AMSJ (FIGURA 2). Estas viviendas son representativas del conjunto ya que simbolizan el $57 \%$ de las viviendas localizadas en DPDU, además, comprenden 14 tipologías con sistemas constructivos y características espaciales diversas.

\section{RESULTADOS}

\section{DISCUSIÓN TEÓRICA-BIBLIOGRÁFICA SOBRE LA FLEXIBILIDAD EN VIVIENDAS}

Actualmente, los aspectos conceptuales asociados a la vivienda son diversos, entre los más representativos se encuentran los de vivienda flexible, vivienda adaptable, vivienda perfectible, vivienda loft, vivienda desjerarquizada, vivienda transformable, vivienda ampliable, vivienda cáscara, vivienda semilla, vivienda dispersa, vivienda divisible, entre otros (Morales Soler et al., 2013). Estas diferentes configuraciones arquitectónicas entienden que las viviendas sufren cambios en su diseño original, su organización espacial y tamaño por su condición inherentemente progresiva (Muñoz Lin, 2014). En TABLA 1 se observa que, mas allá del período temporal, las definiciones de vivienda son similares, predominando la idea de vivienda como proceso, como espacio y como hábitat.

Esta conceptualización de vivienda es compartida mundialmente (TABLA 2). Por ejemplo, en México las viviendas galpón de Carlos González Lobo, en las viviendas incrementales del grupo Chileno Elemental y en las viviendas como proceso progresivo del Centro Experimental de la Vivienda Económica (CEVE) en Argentina. También en Perú, con el 
Tabla 1. Catálogo de definiciones de vivienda (Fuente: elaboración propia, 2021)

\begin{tabular}{|c|c|}
\hline Vivienda como & Autor \\
\hline Proceso; habitacional, dinámico, abierto, evolutivo, adaptable e indeterminado & $\begin{array}{l}\text { Cubillos González (2012), Gutiérrrez Mozo y Pérez del Hoyo (2015), Martín } \\
\text { López (2016) }\end{array}$ \\
\hline $\begin{array}{l}\text { Espacio; de desarrollo y representación de la familia dinámica, adaptativa a las } \\
\text { necesidades y a la variabilidad de la vida }\end{array}$ & $\begin{array}{l}\text { Montaner et al. (201 1), Grounds (2012), Gelabert Abreu y González Couret } \\
\text { (2013), Laiton Suárez (2017), De Paris y Lopes (2018) }\end{array}$ \\
\hline $\begin{array}{l}\text { Hábitat; dinámico, multidimensional, activo, flexible, en constante } \\
\text { transformación }\end{array}$ & $\begin{array}{l}\text { Brandão y Heineck (2003), Agyefi-Mensah (2013), Estaii (2017), Serrano Macías } \\
\text { (2018), Habraken (2020) }\end{array}$ \\
\hline
\end{tabular}

Tabla 2. Conceptos principales de la revisión biblográfica (Fuente: elaboración propia, 2021)

\begin{tabular}{|c|c|}
\hline Concepto principal & Autor \\
\hline Flexibilidad & $\begin{array}{l}\text { Acharya (2013), Aguilar Hidalgo (2013), Agyefi-Mensah (2013), Brandão y Heineck (2003), Cubillos González } \\
\text { (2012), De Paris y Lopes (2018), Estaji (2017), Gelabert Abreu y González Couret (2013), Gilani y Türker } \\
\text { (2020), Gutiérrrez Mozo y Pérez del Hoyo (2015), Grounds (2012), Kumar Dhar et al. (2013), Laiton Suárez } \\
\text { (2017), Mahdinejad y Ehsani Oskovei (2016), Montaner et al. (201 1), Morales Soler et al. (2013), Rian (2011), } \\
\text { Sinclair et al. (2012), Till y Schneider (2005), Trovato (2009). }\end{array}$ \\
\hline Adaptabilidad & $\begin{array}{l}\text { Askar et al. (2021), Aziz et al. (2020), Bezos Alonso (2018), Fiscarelli (2018), French (2016), Friedman (2002), } \\
\text { Jakšić et al. (2017). }\end{array}$ \\
\hline Vivienda progresiva & Lucas et al. (2012), Muñoz Lin (2014). \\
\hline Vivienda semilla & Berretta (1999), Serrano Macías (2018). \\
\hline Edificio abierto & Habraken (2020), Minami (2016). \\
\hline Vivienda incremental & Adler et al. (2018), Gibson y Kendall (2011). \\
\hline Vivienda núcleo, evolutiva, galpón y crecedera & González Lobo (1998), Martín López (2016). \\
\hline
\end{tabular}

Proyecto Experimental de Vivienda (PREVI), las viviendas sociales de Ivoti en Brasil, las viviendas evolutivas de Uruguay, en Colombia las viviendas como un proceso evolutivo y adaptable y las viviendas progresivas en Cuba. En Estados Unidos Friedman y su estudio de adaptabilidad en la vivienda y el Open Building reconocido internacionalmente, etc. (González Lobo, 1998; Adler et al., 2018; Berretta, 1999; Gutiérrrez Mozo y Pérez del Hoyo, 2015; Friedman, 2002; Habraken, 2020).

En el resto de los continentes, los modelos conceptuales de la vivienda se reiteran. En España el trabajo Habitar el Presente, en
India las viviendas de diseño transformable, en Irán la flexibilidad en viviendas, en Rusia vinculan la idea de adaptabilidad con la sostenibilidad, Noruega y las viviendas flexibles, en Japón el concepto de edificio abierto o el trabajo de viviendas ampliadas. En Irak la adaptabilidad en viviendas como herramienta de diseño sostenible, Nueva Zelanda y las viviendas flexibles, las viviendas adaptables en Inglaterra, en Austria se plantea que la vivienda es un sistema de actividad, Alemania y la vivienda cambiante, la vivienda como entidad transformable en el tiempo en los Países Bajos, Portugal y las nociones de flexibilidad residencial, etc. (Montaner et al., 2011 ; Rian,
2011 ; Mahdinejad y Ehsani Oskovei, 2016; Jakšić et al., 2017; Acharya, 2013; Minami, 2016; Aziz et al., 2020; Grounds, 2012; French, 2016; Estaii, 2017; Schneider y Till, 2005; Agyefi-Mensah, 2013; Kumar Dhar et al., 2013).

Son extensas las investigaciones que analizan las causas de las trasformaciones en la vivienda. En su mayoría los trabajos coinciden en que el primer motivo de crecimiento es asumir un núcleo familiar tipo con iguales necesidades y requerimientos, para cualquier lugar geográfico (Rian, 2011; Trovato, 2009), cuando existe una lógica de crecimiento vinculada especialmente con el desarrollo familiar (Rodríguez et al., 
2018). El nivel económico y educativo es motivo de ampliación de las viviendas (Muñoz Lin, 2014; Brandão y Heineck, 2003) al igual que parámetros de funcionalidad, confort y la obsolescencia física (Mahdinejad y Ehsani Oskovei, 2016).

Diversos autores han analizado y desarrollado el concepto de flexibilidad, asociados a las diferentes formas de adecuar el espacio arquitectónico. La flexibilidad es la cualidad de la vivienda de ser susceptible a cambios a lo largo del tiempo (Martín López, 2016; Mahdinejad y Ehsani Oskovei, 2016), siendo el recurso que genera el crecimiento progresivo en las mismas (Laiton Suárez, 2017) y le brinda al usuario la opción de cómo quiere usar los espacios en lugar de predeterminar arquitectónicamente su vida. Diseñar de esta forma tiene beneficios sociales y tecnológicos (Grounds, 2012), entre ellos, los edificios podrían permanecer en uso por más tiempo y podrían ajustarse mejor a su propósito (Acharya, 2013).

El concepto de flexibilidad, ha sido clasificado como inicial (cuando se da en la ocupación inaugural de los espacios) o flexibilidad continua (cuando se da durante el uso, explotación y transformación de la vivienda) (Brandão y Heineck, 2003), Aguilar Hidalgo (2013) y Gelabert Abreu y González Couret (2013) comparten esta clasificación. Gilani y Türker (2020) consideran que la flexibilidad se desfragmenta en flexibilidad estructural, funcional y cultural. También se la define, como flexibilidad espacial (capacidad de cambio en la estructura espacial de un edificio), flexibilidad funcional (capacidad del relleno para permitir que diferentes funciones se desplieguen) y flexibilidad estética (capacidad de alterar la identidad del edificio) (Sinclair et al., 2012).

El análisis teórico-bibliográfico manifiesta que en las últimas dos décadas el concepto de vivienda flexible ha sido estudiado a escala global. No obstante, en términos geográficos la tipología de casa crecedera, cáscara o casa galpón responde a una demanda de vivienda

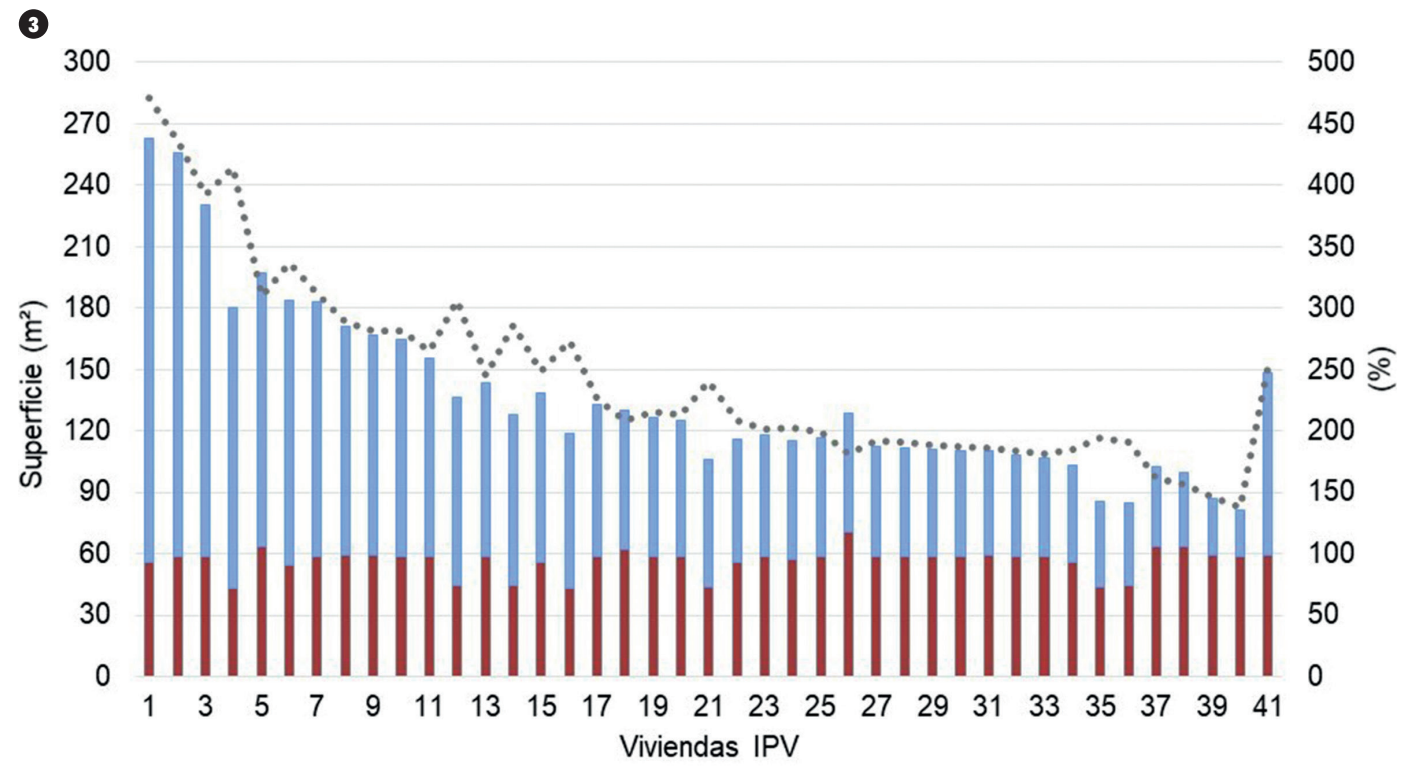

Sup. Original $\left(\mathrm{m}^{2}\right)$

Sup. Ampliada $\left(\mathrm{m}^{2}\right)$

...... Crecimiento $(\%)$

social económica, presente mayormente en países en vías de desarrollo, donde el objetivo es garantizar la adaptación de la vivienda a la estructura variable de la familia y su evolución temporal. En Europa o América del Norte se encuentran otros tipos de crecimiento como la modalidad de cáscara y soporte, que favorecen un mejor aprovechamiento del suelo, con sistemas como el de expansión, de carácter experimental y tecnologías avanzadas (Serrano Macías, 2018). En coincidencia, Gelabert Abreu y González Couret (2013) sostienen que el diseño y construcción de viviendas progresivas tienen enfoques y resultados divergentes en los países con diferente nivel de desarrollo.

\section{ANÁLISIS DEL CRECIMIENTO DE VIVIENDAS IPV}

Como resultado del análisis del crecimiento de 41 viviendas se obtiene el porcentaje de ampliación, para ello, se comparó la superficie actual con la original. Del total de viviendas, 16 han ampliado su superficie entre un $138 \%$ y $199 \%, 18$ entre un $203 \%$ y $289 \%$ y 7 viviendas IPV entre el $312 \%$ y $471 \%$. Como se observa en Figura 3, aquellos prototipos con menos superficie original son los que disponen de mayor superficie de ampliación.

Las tres tipologías que han sufrido mayores ampliaciones son, en primer lugar, la tipología del barrio Virgen de Andacollo, seguido por las del barrio Jardín Policial y en tercer lugar el barrio CGT Rivadavia. Al analizar los períodos temporales en que las viviendas fueron ampliadas, se observa que, la vivienda que se amplió en menor tiempo fue 2 años después de entregada. En su opuesto, la vivienda que tardó más en ampliar lo hizo a los 26 años. Además, el promedio indica que la mayor parte de las viviendas se ampliaron entre los 13 y 14 años (Figura 4).

Del total de viviendas, 13 incorporaron un segundo piso sobre la planta baja (PB), en el que se ubicaron los dormitorios y el baño. En casos puntuales (2 viviendas) la planta alta (PA) se utilizó como depósito o como sala de estudio (2 viviendas). Además, 9 viviendas incluyeron balcón o terraza (FIGURAs 5 y 6). En referencia a la existencia de patios internos, solo el $32 \%$ de las viviendas los incorporó en los procesos de 
4. Períodos de ampliación en viviendas. Fuente: elaboración propia, 2021.

5. Viviendas en PA, con y sin balcón. Fuente: elaboración propia, 2021.

(4)

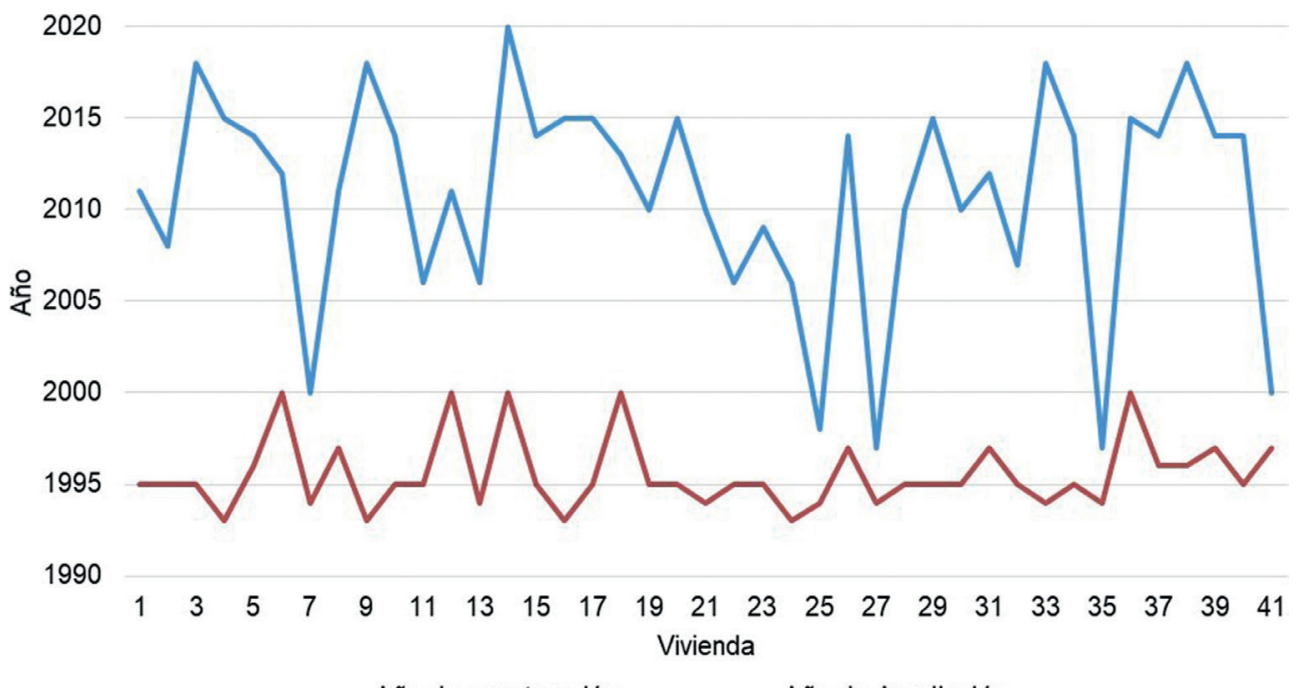

(6

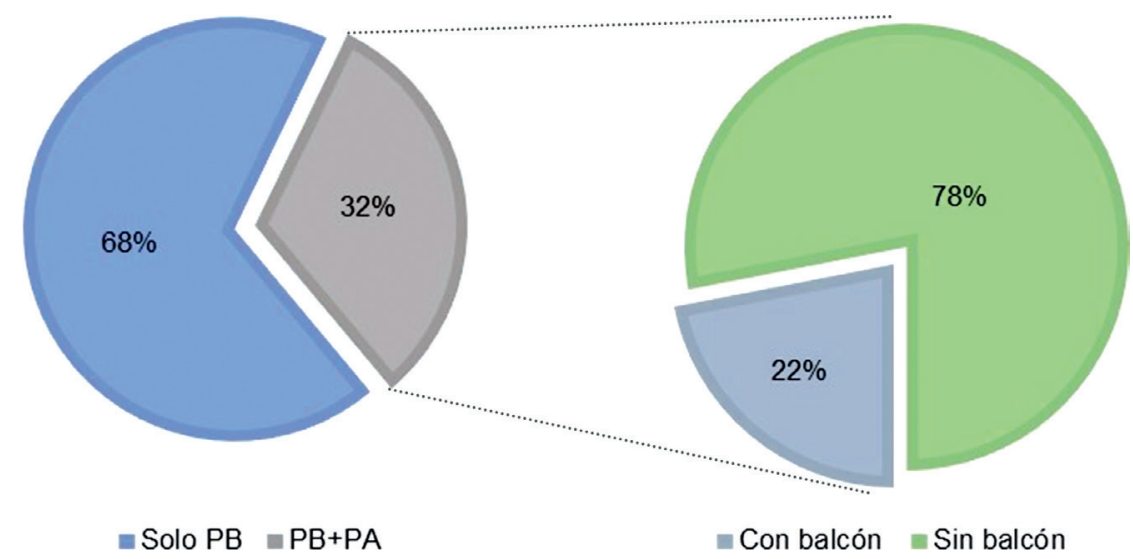

crecimiento, principalmente para la ventilación de habitaciones, el estar y el comedor. Las superficies de los mismos varían entre $4,5 \mathrm{~m}^{2}$ y $10,5 \mathrm{~m}^{2}$, mientras que el promedio es de $6,74 \mathrm{~m}^{2}$.

Por otra parte, con las ampliaciones, el factor de ocupación del terreno (FOT), que es la relación del total de superficie construida y la superficie total de la parcela, se han incrementado entre un $138 \%$ y $470 \%$. En 16 viviendas aumentó entre $138 \%$ y $199 \%$, en 17 entre $200 \%$ y $288 \%$, en 5 el aumento fue entre $306 \%$ y $392 \%$ y, por último, 3 viviendas incrementaron el FOT entre ampliados hacia los laterales (15 viviendas), en segundo lugar, se han ampliado hacia el frente-fondo (1 4 viviendas) y 12 viviendas se han ampliado de forma equitativa hacia los laterales y hacia el frente-fondo (FIgURA 6).

Respecto al cambio de uso en los espacios existentes, 2 viviendas cambiaron la funcionalidad de un dormitorio, por una sala de planchado y por un vestidor respectivamente y en otro caso, el comedor se transformó en oficina. Referido a la incorporación de usos no previstos en el proyecto original, 7 viviendas agregaron un sector de estudio, con escritorio, mientras que en 2 viviendas se construyó un salón para la actividad comercial en la parte frontal de la misma. En base a esto, se identifica que el $27 \%$ de viviendas cambiaron de uso o incorporaron alguno no previsto originalmente. Además, un $27 \%$ de viviendas incorporaron un dormitorio y un $44 \%$ incorporaron uno o dos baños. Por otra parte, si bien la totalidad de las viviendas cuenta con espacio de cochera, se detectó que en un $68 \%$ ese espacio fue techado y cerrado perimetralmente (FIGURA 7).

Asimismo, se observó que en la mitad de viviendas $(51 \%)$ realizaron una construcción al final del terreno y separada de la vivienda, que mayormente es utilizada como depósito. Por otra parte, los espacios con mayores modificaciones son el estar, seguido por el comedor y la cocina. En el $61 \%$ de las viviendas estos espacios fueron ampliados, mientras que el 19\% construyó un espacio nuevo para alguno de estos usos. Finalmente, el 15\% mantuvo los espacios intactos (FIGURA 7).

$47 \%$ y $470 \%$. Además, el FOT promedio de 41 viviendas en su estado original era de 0.2, mientras que el promedio actual (luego de las ampliaciones) es de 0.48 .

Asimismo, en su estado original, 31 viviendas se encontraban adosadas a otra vivienda en un lado del terreno, mientras que el resto (10 viviendas) estaban aisladas en el centro de la parcela. Luego de los procesos de crecimiento, las 41 viviendas se encuentran adosadas, el $78 \%$ de ellas hacia dos laterales del terreno y un $22 \%$ hacia un solo lado del mismo. En referencia a los retiros, la mayoría de las viviendas se han
Referido al aspecto tecnológico-constructivo, de las 11 tipologías originales, todos los muros fueron construidos de ladrillón, 10 tenían muros de $20 \mathrm{~cm}$ de espesor y en una tipología eran de $12 \mathrm{~cm}$. Del análisis de las 41 viviendas se llega a que, al ampliarse las viviendas, esto se mantuvo, tanto en materialidad como en espesor, salvo en 3 viviendas donde los muros son de $30 \mathrm{~cm}$. Además, los muros internos en su mayoría son de $10 \mathrm{~cm}$. y en algunos casos se utilizó tabiques de yeso para las divisiones. 
6. Tipología original y su crecimiento en PB y PA. Fuente: elaboración propia, 2021.

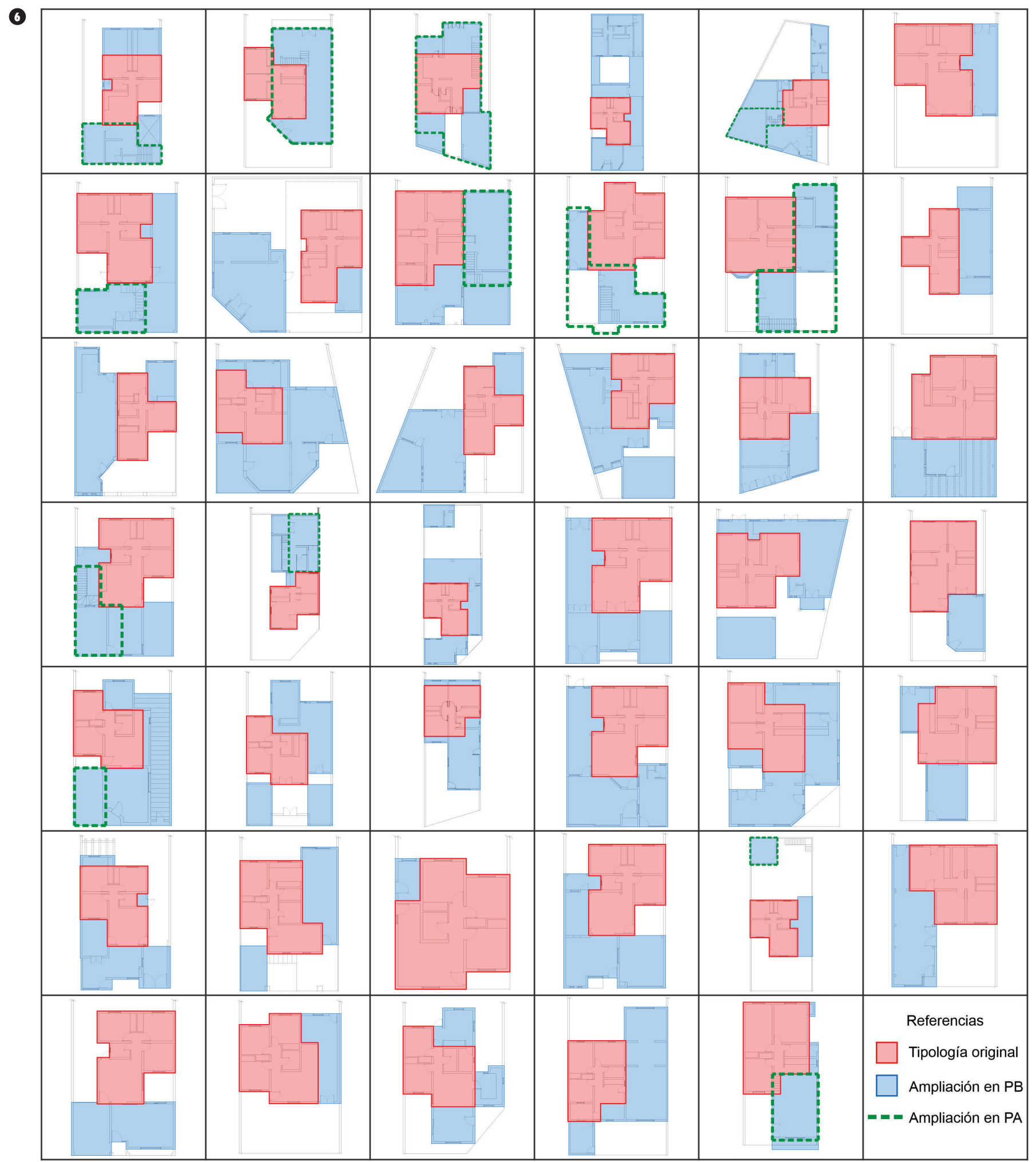


7. Ampliación e incorporación de usos, zonas sanitarias, de descanso y dormitorios. Fuente: elaboración propia, 2021.

(

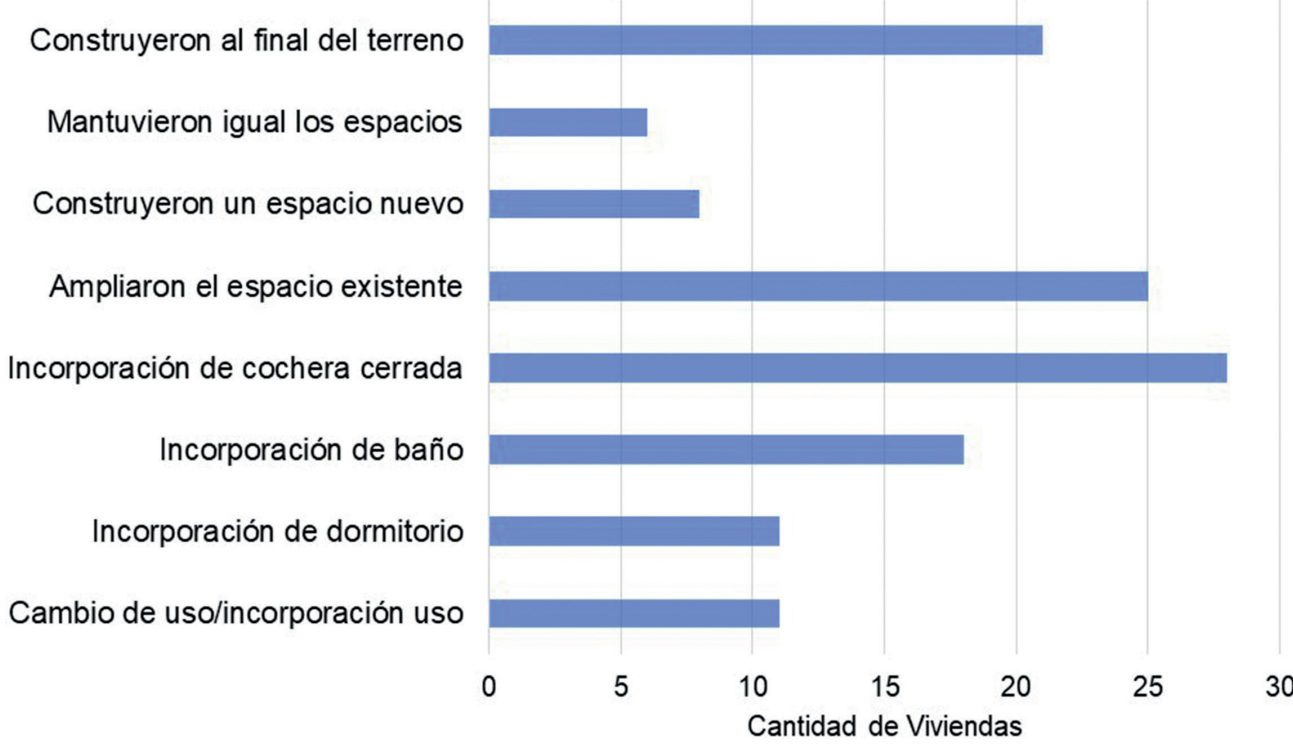

Por su parte, en la cubierta de techo las ampliaciones se realizaron primeramente con ladrillo cerámico y viguetas, en segundo lugar, predomina el hormigón armado $\left(\mathrm{H}^{\circ} \mathrm{A}^{\circ}\right)$ y, por último, la cubierta de tejas. Con respecto a las aberturas (puertas y ventanas), predomina la carpintería metálica de chapa doblada, en segundo lugar, la de madera y, por último, el aluminio. En cuanto a las escaleras, el $70 \%$ son metálicas, seguidas por las de $\mathrm{H}^{\circ} \mathrm{A}^{\circ}$. Pérez-Pérez (2016), sostiene que es sustancial incorporar tecnologías que articulen los sistemas que integran la vivienda (como el estructural y las instalaciones) y faciliten su evolución según los requisitos del usuario.

\section{DISCUSIÓN Y CONCLUSIONES}

La investigación teórica-bibliográfica sobre la flexibilidad en la vivienda ha permitido advertir, que los casos de estudio analizados han seguido los patrones expuestos por Cubillos González (2012) y según la clasificación del mismo autor, se han observado con mayor preponderancia las modificaciones internas que externas. Siguiendo los conceptos de Brandão y Heineck (2003), Aguilar Hidalgo (2013) y Gelabert Abreu y González Couret (2013) se observó mayor flexibilidad continua que inicial. Asimismo, en base a las clasificaciones de Gilani y Türker (2020) y Sinclair et al. (2012) la flexibilidad funcional es la predominante en las modificaciones de las viviendas, seguida por la flexibilidad estética.

En la misma línea, las características de crecimiento predominante es la ampliación hacia los laterales y frente de la vivienda (incluso en los prototipos con PA), dejando disponible la parte posterior del terreno, lo que evidencia la necesidad mantenida durante la fase de uso, de contar con un espacio al aire libre de carácter privado. Asimismo, no se encontró relación o patrón común, entre la tipología arquitectónica y la existencia de un segundo piso, la presencia de patios interiores o el incremento del FOT lo que muestra que las formas de crecimiento responden a necesidades particulares del usuario (dan cuenta de lo general).

Al comparar las viviendas originales y las actuales se observa que todas han incrementado la superficie cubierta para incorporar usos específicos que no fueron originalmente tenidos en cuenta por el IPV. Para las tipologías arquitectónicas que forman parte de los casos de estudio, se asevera la necesidad de incrementar las dimensiones de los lugares de estar y comedor, incluir un espacio de depósito y guardado e incorporar un espacio de estudio. Además, se considera necesario integrar la cochera a la vivienda como un espacio cubierto y cuidar la proporción entre edificación y superficie de terreno, que como se ha observado, se ha incrementado de forma exponencial desde el inicio de la fase de uso.

Como trabajos futuros se pretende realizar encuestas que permitan precisar los requerimientos de los residentes en relación a las posibilidades de adaptación y ampliación de las viviendas IPV en el AMSJ. Seguidamente se pretende abordar la temática en cuestión, pero en viviendas donde la construcción de ampliaciones sea de manera informal o mediante autoconstrucción.

\section{AGRADECIMIENTOS}

Se agradece al Consejo Nacional de Investigaciones Científicas y Técnicas, por el financiamiento de tesis doctoral en curso de la autora, de la cual este artículo es producto. Se agradece también a la Facultad de Arquitectura, Urbanismo y Diseño y a la Universidad Nacional de San Juan.

\section{REFERENCIAS BIBLIOGRÁFICAS}

Acharya, L. (2013). Flexible architechture for the dynamic societies: reflection on a journey from the 20th Century into the future. [Tesis de maestría, University of Tromsø] https://munin.vit.no/ handle/10037/5462? locale-attribute $=$ en .

Adler, V., Vera, F., Wainer, L. S., Roquero, P., Poskus, M. A., Valenzuela, L., Letelier, M., Olivares, P., Treimun, J., Gamboa, A., Canales, K., Guajardo, J., Libertun de Duren, N., Davis, D.E., Donovan, M. G., Claramunt Torche, P., y Silva, M. P. (2018). Vivienda ¿̇Qué viene? De pensar la unidad a construir ciudad. Banco Interamericano de Desarrollo, Vol. 659

Aguilar Hidalgo, C. A. (2013). Arquitectura Polivalente. Proyecto arquitectónico de conjunto 
habitacional adaptable hacia la sustentabilidad en Comitán, Chiapas. 01-08. https://www. academia.edu/8099594/Arquitectura_Polivalente Agyefi-Mensah, S. (2013). Functionality and adaptability of design solutions for public apartment buildings in Ghana: towards evidencebased design for sustainable lifespan building performance. [Tesis doctoral, Technische Universiteit Eindhoven] https://doi.org/10.6100/ IR762203

Askar, R., Bragança, L., y Gervásio, H. (2021). Adaptability of Buildings: A Critical Review on the Concept Evolution. Applied Sciences, 17 (10), 4483. https://doi.org/10.3390/appl 1104483

Aziz, S. S., Alobaydi, D., y Salih, A. B. (15 de abril de 2020). Studying Flexibility and Adaptability as Key Sustainable Measures for Spaces in Dwelling Units: A Case Study in Baghdad. $3^{\circ}$ International Conference on Sustainable Engineering Techniques, Baghdad, Iraq. 881 (1), 012-019. https://doi.org/10.1088/1757899X/881/1/012019

Berretta, H. (1999). Sistema Constructivo Semilla. Estudios del Hábitat, 2(6), 65-74. https://www.fau.unlp.edu.ar/ web2018/wp-content/uploads/2018/10/ estudiosdelhabitatvol6HoracioBerretta.pdf.pdf

Bezos Alonso, J. L. (2018). El concepto de Low Road de Stewart Brand como fundamento de estrategias para la adaptabilidad de los espacios en la vivienda contemporánea. Proyecto, Progreso, Arquitectura, (19), 56-69. https://doi. org/10.12795/ppa.2018.i19.03

Brandão, D., y Heineck, L. F. (2003). Significado multidimensional e dinâmico do morar: compreendendo as modificações na fase de uso e propondo flexibilidade nas habitações sociais. Ambiente Construído, 3(4), 35-48. https://seer.ufrgs.br/ambienteconstruido/article/ view/3504/1905

Camargo Sierra, A. P. (2019). Vivienda y estrategias familiares de vida en barrios populares consolidados en Bogotá. Revista INVI, 35(98), 101 - 125. https://doi.org/10.4067/S071883582020000100101

Cubillos González, R. A. (2012). Estudio y gestión de estándares mínimos de flexibilidad en la vivienda social en Bogotá. Revista de Arquitectura (Bogotá), 14(1), 65-75. https:// revistadearquitectura.ucatolica.edu.co/article/ view/727

De Paris, S., y Lopes, C. (2018). Housing flexibility problem: Review of recent limitations and solutions. Frontiers of Architectural Research, 7(1), 80-91. https://doi.org/10.1016/i. foar.2017.11.004

Estaji, H. (2017). A Review of Flexibility and Adaptability in Housing Design. International Journal of Contemporary Architecture The New ARCH, 4(2), 37-49. https://doi.org/10.14621/ tna.20170204

Fiscarelli, D. (2018). Aspectos cualitativos de la vivienda de interés social: Aproximaciones teóricas desde la complejidad del proyecto. Revista Arquisur, 8(13), 1-12. https://doi. org/10.14409/ar.v8i13.6817

French, H. (2016). Adaptable Housing: accommodating change. [Tesis doctoral, University of Brighton] https://research.brighton. ac.uk/en/studentTheses/adaptable-housingaccommodating-change

Friedman, A. (2002). The Adaptable House: Designing Homes for Change. McGraw-Hill Professional.

Gelabert Abreu, D. y González Couret, D. (2013). Vivienda progresiva y flexible. Aprendiendo del repertorio. Arquitectura y Urbanismo, 34(2), 48 63. Recuperado en 19 de noviembre de 2021, de http://scielo.sld.cu/scielo.php? script $=$ sci arttext\&pid $=$ S1815-58982013000200005\&lng $=\mathrm{es} \&$ tlng $=\mathrm{es}$.

Gibson, M. D., y Kendall, S. H. (15-17 de noviembre de 2011 ). Architecture in the Fourth Dimension. Methods and Practices for a Sustainable Building Stock. Conference of CIB W104 and W1 10. International Council for Research and Innovation in Building and Construction, Boston, Massachusetts, USA. https://doi.org/10.2752/14 $7800411 \times 12858412044357$

Gilani, G., y Türker, Ö. O. (2020). Assessing flexibility in real estate mass housing. Revista Arquitectura, 16(1), 154-175. https://doi.org/10.4013/ arq.2020.161.09

González Lobo, C. (1998). Vivienda y ciudad posibles. Escala.

Grounds, M. (2012). Variations: Flexibility and Adaptability in Medium Density Housing. [Tesis de maestría, Unitec Institute of Technology]. https:// www.researchbank.ac.nz/handle/10652/2075
Gutiérrrez Mozo, M. E., y Pérez del Hoyo, R. (2015). PREVI: la tipología entre la fluidez de la vida y el rigor de la edificación. Cuadernos de Vivienda y Urbanismo, 8(16), 186-205. https://doi. org/10.11144/Javeriana.cvu8-16.ptfv

Habraken, N. J. (2020). Open Building; brief introduction. https://www.habraken.com/html/ introduction.htm

Instituto de Investigaciones Económicas y Estadísticas (2020). Proyecciones de viviendas 2020. https:// web.sanjuan.gob.ar/iiee/

IRAM. (1969). Norma IRAM 11553. Durabilidad de los edificios. Argentina.

Jakšić, Ž., Trivunić, M., y Adamtsevich, A. (2017). Flexibility and adaptability - key elements of end-user participation in living space designing. MATEC Web of Conferences 106, 1-10. https:// doi.org/10.1051/matecconf/201710601001

Kumar Dhar, T., Sk. Maruf Hossain, M., y Rubayet Rahaman, K. (2013). How does flexible design promote resource efficiency for housing? A study of Khulna, Bangladesh. Smart and Sustainable Built Environment, 2(2), 140-157. http://doi. org/10.1 108/SASBE-10-2012-0051

Laiton Suárez, M. P. (2017). Prototipos flexibles. Proyecto habitacional en el barrio popular Buenos Aires (Soacha). Revista de Arquitectura, 19(1), 70-85. https://doi.org/10.14718/ RevArq.2017.19.1.1271

Lucas, P., Salas, J., y Barrionuevo, R. (2012). Cuarenta años del PREVI-LIMA: Algunas enseñanzas para la industrialización de la vivienda de bajo coste en Latinoamérica. Informes de la Construcción, 64(525), 51-62. https://doi.org/10.3989/ ic. 11.024

Mahdinejad, J., y Ehsani Oskouei, S. F. (2016). The Necessity of Flexibility with Regard To User' s Satisfaction in Multifunctional Buildings of Traditional Architecture of Iran (Case Study: The Home-Hussainia of Aminiha). Mediterranean Journal of Social Sciences, 7(4 S1) https://doi.org/10.5901/miss.2016. v7n4S1p133

Martín López, L. (2016). El crecimiento programado en tres escalas: el caso de las viviendas NWwKT. Bitácora Arquitectura, 32, 4-13. http://doi. org/10.22201/fa.14058901p.2016.32.56188

Minami, K. (2016). The efforts to develop longer life housing with adaptability in Japan. 
Energy Procedia, 96, 662-673. https://doi. org/10.1016/i.egypro.2016.09.124

Montaner, J. M., Muxí, Z., y Falagán, D. H. (2011). Herramientas para habitar el presente: la vivienda del siglo XXI. Máster Laboratorio de la vivienda del siglo XXI.

Morales Soler, E., Alonso Mallen, R., y Moreno Cruz, E. (2013). La vivienda como proceso. Estrategias de flexibilidad. Hábitat y Sociedad, 4, 33-54. https://idus.us.es/bitstream/ handle/1 1441/22157/file_1. pdf? sequence $=1$

Muñoz Lin, T. (2014). Evolución del espacio doméstico en "blocks" de vivienda social. Autoconstrucción y vulnerabilidad en conjuntos de vivienda básica. Revista CIS,9 (15), 3-26. https://dialnet.unirioja. es/servlet/articulo? codigo $=6310269$

Pérez-Pérez, A. L. (2016). El diseño de la vivienda de interés social. La satisfacción de las necesidades y expectativas del usuario. Revista de Arquitectura, 18(1), 67-75. https://doi.org/10.14718/ RevArq.2016.18.1.7

Petrović, B.; Zhang, X.; Eriksson, O.; Wallhagen, M. (2021) Life Cycle Cost Analysis of a Single-Family House in Sweden. Buildings, 17 (5), 215. https:// doi.org/10.3390/buildings 11050215
Rian, I. (5-7 de diciembre de 201 1). Multicultural Flexible Housing: addressing the need and scope of flexible housing in cosmopolitan indian cities. $5^{\circ}$ Conference International Conference y Workshop on Built Environment in Developing Countries, Penang, Malaysia.

Rodríguez, M. C., Rodríguez, M. F., y Zapata, M.C. (2018). Mercantilización y expansión de la inquilinización informal en villas de Buenos Aires, Argentina. Revista INVI, 33(93), 125-150. https:// doi.org/10.4067/S0718-83582018000200125

Schneider, T., y Till, J. (2005). Flexible housing: Opportunities and limits. Architectural Research Quarterly, 9(2), 157-166. https://doi. org/10.1017/S1359135505000199

Secretaría de Vivienda. (2019). Estándares mínimos de calidad para viviendas de interés social. Marco para la promoción de viviendas inclusivas, asequibles y sostenibles. Plan Nacional de Viviendas. https://www.argentina.gob.ar/sites/ default/files/if-2019-72275570-apn-dnasyfmi.pdf Serrano Macías, A. (2018). Vivienda Crecedera. Prototipo para la regeneración urbana en Valle del Paraiso [Tesis de grado, Universidad Politécnica de Madrid] http://oa.upm.es/51702/
Sinclair, B., Mousazadeh, S., y Safarzadeh, G.

(2012). Agility, Adaptability + Appropriateness: Conceiving, Crafting y Constructing an Architecture of the 21 st Century. ARCC Journal for Architectural Research, 9(1). https://doi. org/10.17831/enq:arcc.v9i1.65

Teles, R., y González, M. S. (2013). Ampliaciones en viviendas sociales - Estudio de caso en Ivoti Brasil. Revista Ingenieria de Construccion, 28(3), 237-250. https://doi.org/10.4067/s071850732013000300002

Till, J., y Schneider, T. (2005). Flexible housing: the means to the end. Architectural Research Quarterly 9(3-4), 287-296 https://doi. org/10.1017/S1359135505000345

Trovato, G. (2009). Definición de ámbitos de flexibilidad para una vivienda versátil, perfectible, móvil y ampliable. Ciudad y Territorio, Estudios Territoriales, XLI (161-16),599-614. ISSN 1133 4762.

Ziemski, Jarosław. (2018). The use of LCC method in running cost analysis of a single-family house. Economic and Environmental Studies, 18(1), 445 455. https://doi.org/10.25167/ees.2018.45.25 